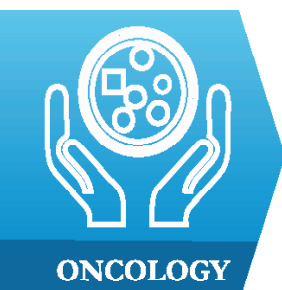

1) Department of Morphological Sciences, Iuliu Hatieganu University of Medicine and Pharmacy, ClujNapoca, Romania

2) Department of Endocrinology and Nuclear Medicine, "Prof. Dr. Ion Chiricuta" Institute of Oncology, Cluj-Napoca, Romania
DOI: $10.15386 / \mathrm{mpr}-1341$

Manuscript received: 13.04.2019

Accepted: 20.06.2019

Address for correspondence:

badan_marius@yahoo.com

\section{A review on immunohistochemical and histopathologic validation in PET-CT findings with consideration to microRNAs}

Marius-Ioan Bădan ${ }^{1}$, Eduard-Alexandru Bonci ${ }^{1}$, Doina Piciu ${ }^{1,2}$

\begin{abstract}
Purpose. This review provides an overview of some of the most recent clinical trials which investigated various types of cancer and other diseases, through the use of PET-CT imaging, highlighting the use of immunohistochemical stains or conventional histopathology for the validation or contradiction of their hypothesis. Furthermore, we investigate a potential new direction of research by analyzing the upcoming role of microRNAs in disease confirmation.

Methods. An extensive search of MEDLINE/ PubMed and SCOPUS electronic databases was made, using the MeSH terms "positron emission tomography computed tomography" and "immunohistochemistry" as well as "SUV" and "immunohistochemistry", restricting the search by clinical trials and time period. Further searches were made for articles regarding Ki-67 and microRNAs in correlation with metabolic PET-CT uptake.
\end{abstract}

Results. Out of all 389 initial search results, 27 original articles were found relevant to the topic. Their contents were synthesized and discussed regarding the matter at hand. No relevant clinical trials involving microRNAs were found.

Conclusions. Immunohistochemical and histopathologic results remain widely used and indispensable in modern research, concerning PET-CT validation. Possible candidates for diagnosis confirmation, in future research, may reside in the further development of microRNAs.

Keywords: histopathology, immunohistochemistry, Positron Emission Tomography Computed Tomography, microRNAs, neoplasms

\section{Introduction}

Modern medicine is detaching itself from conventional histopathologic "gold standard" practices, with the utilization of computed tomography and magnetic resonance imaging, coupled with a range of clinical laboratory tests that are becoming ubiquitous, increasing its diagnostic insight in various diseases. Nevertheless, as technology edges forward, we find ourselves at a point where histopathologic and immunohistochemical validation in the diagnostic procedure, more so in medical research fields, still have a strong grip in confirming and understanding pathological processes, as will be discussed in this paper.
From a clinical standpoint of view, radiotherapists and oncologists still await, at present, on pathologists for assistance in tumor diagnosis. The pathologist finds himself in a pivotal point between disease, therapy, and prognosis. Correspondingly important are histopathologic confirmations involving test protocols and efficacy to novel therapies in clinical trials and current research.

Positron emission tomography computed tomography (PET-CT) hybrid imaging provides important key aspects of tumor topography, as well as from a functional viewpoint, using radiolabeled molecules associated with cell 
metabolism [1]. This relatively new diagnostic procedure is gaining momentum as an instrument for cancer detection, as more and more research is being oriented at describing its potential and limitations in non-invasive cancer depiction, adding to the series of tests set to replace the burdensome need for biopsies. Notwithstanding, it seems that PET-CT hybrid imaging, in its attempts to surpass it, still needs to be held against the current histopathologic standard, as it takes its role in modern medicine.

In this systematic review, we gather evidence supporting the fact that immunohistochemistry (IHC) and histopathology still perform an important role in contemporary medicine and in the characterization of imaging tools, through the process of searching the scientific literature for the most recent clinical trials relevant to this topic. We attempt to characterize this subject by means of an original evaluation, with no current published review exploring the issue at this moment. Additionally, we study and exemplify high-interest research niches surrounding the attempts to portray PET-CT hybrid imaging, through immunohistochemical validation, as a potential candidate for tumor description and prognosis by means of cell proliferation. Furthermore, we assess the emerging potential of microRNAs, highly conserved non-coding RNA molecules involved in the regulation of gene expression, as candidates for future PET-CT capabilities for tumor description.

\section{Methods}

This review adhered, as applicable, to the PRISMA-P 2015 checklist.

\section{Data sources}

An initial search, of MEDLINE/ PubMed indexed and published articles, was made using the term "positron emission tomography computed tomography" and the MeSH terms: "immunohistochemistry" as well as "SUV" and "immunohistochemistry". Results were afterwards restricted by selecting only clinical trials and furthermore restricted to titles only published after the year 2000 up until February 2019.

Furthermore, the electronic database SCOPUS was screened for titles, abstracts, and keywords, using the following search strategy:

- TITLE-ABS-KEY (positron AND emission AND tomography AND computed AND tomography AND immunohistochemistry) DOCTYPE (ar) AND "clinical trial".

\section{Study selection}

Firstly, the articles were screened and duplicates were eliminated. Secondly, the selected articles were rescreened using the following inclusion criteria:

- Published after the year 2000 (a 19 year time period, to maintain relevance to recent findings);

- Use of immunohistochemical or histopathologic validation for PET-CT outcomes or for correlation with a hypothesis regarding IHC expression;

- Immunohistochemical markers used had to be specified.

Articles were not found to be relevant for this review if they:

- Were published before the year 2000;

- If the completion of the study was not sufficiently dependent on IHC or histopathological examinations;

- Contained the aforementioned search terms, without having a direct implication of PET-CT validation by means of immunohistochemical or histopathologic tests;

- Were found to be case reports or reviews (SCOPUS);

- Were not related to the field of medicine (SCOPUS).

The articles were selected regardless of results confirming or contradicting their initial hypothesis. All diseases studied in the trials were considered relevant. Emphasis was given to neoplastic diseases. There were no restrictions imposed on language.

\section{Data extraction}

Out of all 389 initial search results studied, 364 were retained after removing duplicate results. The resulting articles were then screened by two independent researchers following the aforementioned inclusion and exclusion criteria. Disagreements regarding the addition of articles were managed by discussion or by a third opinion. In the end, 27 original articles were found to be relevant to the topic (Figure 1).

All articles were screened for types of radiopharmaceuticals used in PET-CT imaging, objectives, findings and types of IHC markers or histopathological findings used for validation. The data were synthesized and presented for each case.

In addition, for the completion of this review and the evaluation of current trends in cancer diagnostics, the investigators gathered information surrounding the focus at hand, by screening MEDLINE/ PubMed indexed clinical trial articles using the MeSH terms: ("positron emission tomography computed tomography" AND "microARN"). A broader evaluation of the subject was made by also searching for reviews regarding this subject. Furthermore, an ample assessment of indexed articles was oriented at reviews and trials, without strict time limitations, for research meant to correlate PET-CT standardized uptake values (SUV) and immunohistochemical marker expression of cellular proliferation (Ki-67). The resulting articles are reviewed in the discussion section under their appropriate subtitle. 


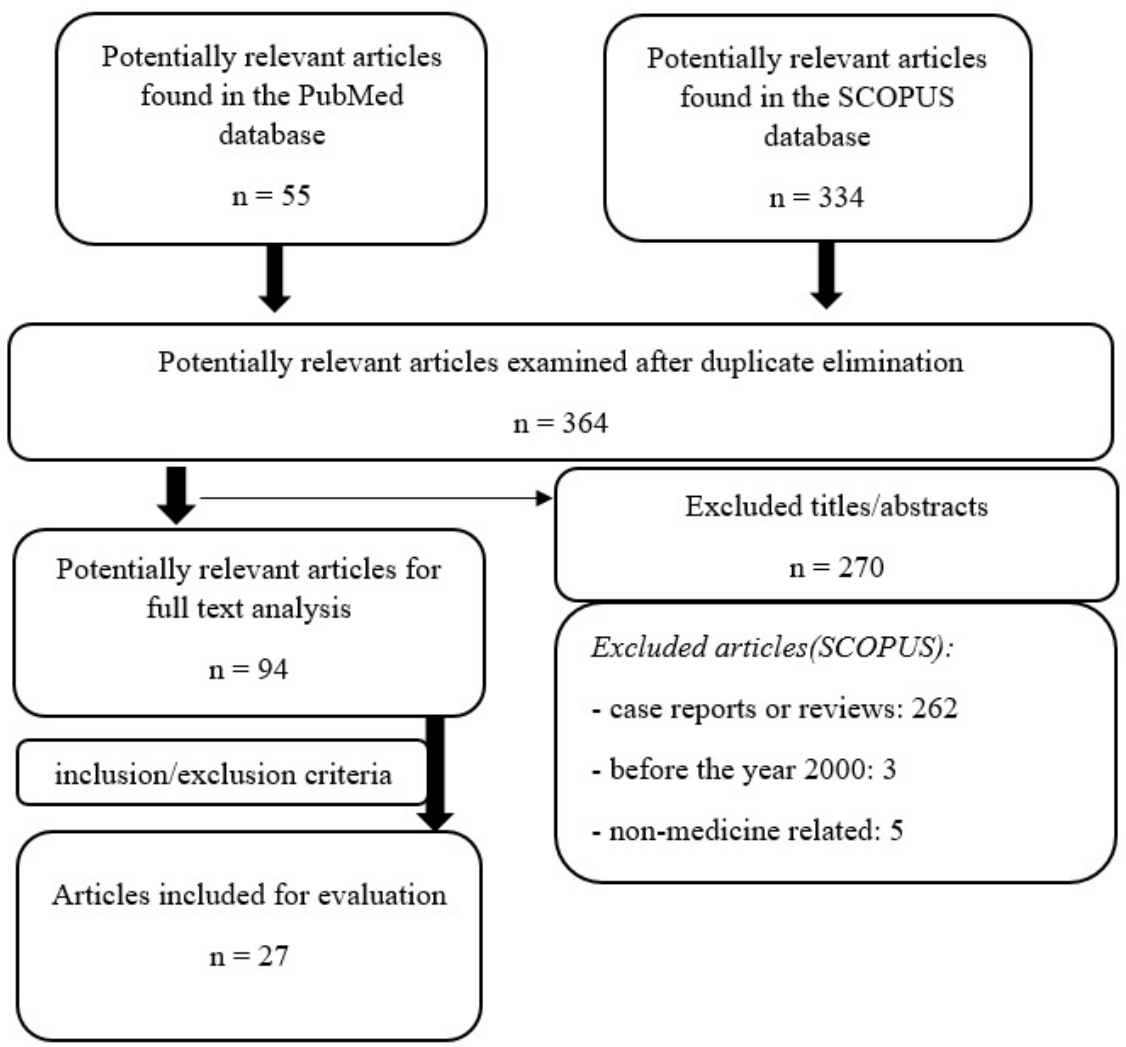

Figure 1. Study selection summary.

\section{Results}

Following an extensive review of content extracted from the 27 selected clinical trials (Table I), data regarding their characteristics can be summarized as follows:

- Twenty-four articles contained research involving neoplastic tumors (of which: 7 were of breast cancer, 8 pulmonary cancers, and 2 colorectal adenocarcinomas);

- Other neoplastic tumors included: lymphoma, thyroid carcinoma, prostate cancer, endometrial carcinoma, head and neck squamous cell carcinoma (HNSCC), insulinoma and mesenchymal uterine tumors;

- The remaining three articles dealt with inflammatory/infectious diseases, neurodegenerative diseases and diseases of blood vessels;

- Twelve trials were involved in detection/ diagnosis, 7 attempted to establish a direct correlation with immunohistochemistry/histopathology, 3 monitored response to treatment and 2 involved the study of tumor angiogenesis;

- Eleven studies used ${ }^{18} \mathrm{~F}$-fluorodeoxyglucose (FDG) as their primary radio-labeled marker, 8 used other substances, and 5 compared FDG to other radio-labeled indicators;
- Immunohistochemical markers varied greatly. Slightly more used were Ki-67 and vascular markers;

- Study populations varied from 4 to 147 patients.

Details of the selected studies, in relationship to their IHC and histopathological results, and aspects of their conclusions are given in the following statements.

Concerning non-neoplastic diseases, immunohistochemical findings, using a CD68 marker for macrophage density and vascular endothelial growth factor, were used for patients undergoing carotid endarterectomy after an initial FDG PET/CT uptake determination. Increased plaque metabolism was demonstrated to correlate with increased inflammation and angiogenesis, whereas the histological and PET markers were shown to be inversely related in plaque calcifications [2]. Modern research practices in neurodegenerative diseases still make use, and demonstrate, the invaluable nature of histopathologic confirmation in this study of $A \beta$ plaques being highlighted by [18F]flutemetamol PET [3]. Immunohistochemical stains were also used, in this small 10 patient study, for confirming sarcoidosis patients, from other inflammatory or infectious lesions who underwent (4S)-4-(3-(18)F-fluoropropyl)-1glutamate ((18)F-FSPG) PET scans [4]. 
Table I. Authors and titles/subjects of selected clinical trials.

\begin{tabular}{|c|c|c|c|}
\hline Investigators & Year & No. of patients & Subject/Issues studied \\
\hline Antwi et al. & 2015 & 5 & > Localization of Hidden Insulinomas with ${ }^{68} \mathrm{Ga}-\mathrm{DOTA}-E x e n d i n-4$ PET/CT: A Pil... \\
\hline Baek et al. & 2012 & 10 & $>$ Exploratory clinical trial for imaging $\mathrm{x}_{\mathrm{c}}^{-}$transporter using positron emission to... \\
\hline Beach et al. & 2016 & 68 & $>$ Detection of Striatal Amyloid Plaques with [18F]flutemetamol: Validation wi... \\
\hline Buck et al. & 2002 & 30 & $>3$-deoxy-3-[(18)F]fluorothymidine-positron emission tomography for noninv... \\
\hline Chae et al. & 2016 & 10 & > Exploratory Clinical Investigation of (4S)-4-(3-18F-Fluoropropyl)-L-Glutamat... \\
\hline Cochet et al. & 2012 & 40 & $>$ Evaluation of breast tumor blood flow with dynamic first-pass 18F-FDG PET]... \\
\hline de Oliveira Costa et al. & 2016 & 147 & $>$ Interim fluorine-18 fluorodeoxyglucose PET-computed tomography and cell... \\
\hline Gebhart et al. & 2016 & 56 & $>$ Molecular imaging as a tool to investigate heterogeneity of advanced HER $2 \ldots$ \\
\hline Gemignani et al. & 2013 & 48 & $>$ Feasibility and predictability of perioperative PET and estrogen receptor liga.. \\
\hline Groves et al. & 2011 & 20 & $>{ }^{18} \mathrm{~F}-\mathrm{FDG}$ PET and biomarkers for tumour angiogenesis in early breast cancer \\
\hline Han et al. & 2009 & 33 & $>$ Correlation of ${ }^{18} \mathrm{~F}$-FDG PET activity with expressions of survivin, Ki67, and CD... \\
\hline Higashi et al. & 2004 & 47 & $>$ P-glycoprotein expression is associated with FDG uptake and cell differentia... \\
\hline Hu et al. & 2013 & 32 & > Hypoxia imaging with 18F-fluoroerythronitroimidazole integrated PET/CT a... \\
\hline Jacob et al. & 2003 & 4 & $>$ Positron emission tomography with $[(18)$ F]FDOPA and $[(18) F] F D G$ in the ima... \\
\hline Jacobs et al. & 2011 & 19 & $>$ Monitoring of neoadjuvant chemotherapy using multiparametric, ${ }^{23} \mathrm{Na}$ sodiu... \\
\hline Kang et al. & 2016 & $21 / 13 *$ & $>$ Comparing the Diagnostic Potential of 68Ga-Alfatide II and 18F-FDG in Diff... \\
\hline Lansoy-Kuhn et al. & 2013 & 26 & $>$ Relationship between the immunohistochemistry of the primary tumour and... \\
\hline Mena et al. & 2012 & 39 & > 11C-Acetate PET/CT in localized prostate cancer: a study with MRI and histo... \\
\hline Menezes et al. & 2011 & 21 & > Investigating vulnerable atheroma using combined (18)F-FDG PET/CT angio... \\
\hline Nakamura et al. & 2010 & 44 & $>$ The SUVmax of 18F-FDG PET correlates with histological grade in endometri... \\
\hline Osborne et al. & 2010 & 36 & $>18 \mathrm{~F}-\mathrm{FDG}$ PET of locally invasive breast cancer and association of estrogen re... \\
\hline Riedl et al. & 2007 & 90 & $>18$ F-FDG PET scanning correlates with tissue markers of poor prognosis and... \\
\hline Tian et al. & 2004 & 19 & $>$ Expression of Glut-1 and Glut-3 in untreated oral squamous cell carcinoma c... \\
\hline Venema et al. & 2017 & 13 & $>$ Androgen and Estrogen Receptor Imaging in Metastatic Breast Cancer Patie... \\
\hline Yamamoto et al. & 2009 & 26 & > Detection of colorectal cancer using 18F-FLT PET: comparison with 1"'F-FDG P... \\
\hline Yang et al. & 2010 & 31 & $>$ Imaging of proliferation with $18 \mathrm{~F}-\mathrm{FLT}$ PET/CT versus $18 \mathrm{~F}-\mathrm{FDG}$ PET/CT in non-... \\
\hline Zhao et al. & 2013 & 47 & $>18 \mathrm{~F}-\mathrm{FES}$ and 18F-FDG PET for differential diagnosis and quantitative evaluati... \\
\hline
\end{tabular}

* comparative study involving 21 patients diagnosed with neoplasia and 13 with lung tuberculosis.

Regarding clinical trials involved in neoplastic diseases, we found that histopathologic diagnosis was necessary for four patients with benign insulinomas who participated in a clinical trial which determined the feasibility of (68)Ga-DOTA-exendin-4 PET/CT insulinoma detection [5]. Attempts at demonstrating a link between 9 immunohistochemical markers in well-differentiated thyroid carcinomas and FDG-PET uptake were made in a recent study, with no significant correlation being established at the end [6]. (11)C-acetate was used in a trial, to demonstrate a higher concentration of the substance in tumor foci, rather than in normal tissue. Validation but also limitations of this finding, in which the method could not differentiate between tumor and benign prostate hyperplastic tissue, were demonstrated through histopathology [7]. In a prospective study concerning 111 patients with diffuse large B-cell nonHodgkin lymphoma, treated with R-CHOP-21 protocols, established a very good prognostic value for patients with negative interim PET (iPET)-computed tomography (CT) after 48 months. Immunohistochemistry was used for categorizing germinal center versus nongerminal center subtypes of diffuse large B-cell non-Hodgkin lymphomas [8]. GLUT-1 and GLUT-3 immunohistological markers were used in another study concerning oral squamous cell carcinoma patients, which showed high FDG uptake in carcinomas with Glut-1 and Glut-3 overexpression [9]. Analyzing SUV values of $16 \alpha-(18) F$-fluoro-17 $\beta$-estradiol ((18)F-FES) and FDG showed a correlation between (18) F-FES uptake and estrogen and progesterone as well as a correlation between FDG and GLUT-1 and Ki-67 in patients with uterine sarcomas out of 47 patients diagnosed 
preoperatively with mesenchymal uterine tumors [10]. Correlation of radiolabeled markers with IHC stains for cell proliferation (Ki-67), was shown in one study, which assessed the comparison between 3 '-deoxy $-3^{\prime}-{ }^{18} \mathrm{~F}$ fluorothymidine (FLT) and FDG in colorectal patients, to not always be significant [11]. Further examination of the selected articles, revealed a trial involving the use of Ki-67, alongside GLUT1 and p53 to demonstrate a correlation with maximum SUV values in 90 patients with liver metastatic resectable colorectal cancer, showing the prognostic value of noninvasive FDG PET scans [12]. Correlation between GLUT-1 expression, in primary endometrial cancers, and FDG-PET uptake was also established [13].

Particular interest in the matter was given, in the last 19 years, to lung neoplasms. Non-small cell lung cancer (NSCLC) patients who underwent (18) F-fluoroerythronitroimidazole ((18)F-FETNIM) PET/ CT examinations revealed the possibility of using (18) F-FETNIM as a marker for hypoxic conditions, which correlated with a worse outcome, demonstrated by the expressions of HIF- $1 \alpha$, GLUT-1, and VEGF, and by using a tumor-to-mediastinum ratio parameter [14]. An interesting correlation between the PET imaging tracer, (18)F-FSPG, and the $\mathrm{xCT}$ subunit of system $\mathrm{x}_{\mathrm{C}}^{-}$, with roles in cancer growth, was indicated in a 10 NSCLC patient trial by means of using IHC for the subunit together with CD44 [15]. A comparison between the diagnostic efficacies of FLT and FDG PET/CT was made for patients with nonsmall-cell lung cancer, assessing therapy response by measuring the radio-labeled marker uptake and correlating it to cyclin D1 labeling index from positive lymph nodes. FLT uptake was shown to have a better correlation with tumor cell proliferation, through the cyclin D1 labeling index expression [16]. Once more FLT and Ki-67 were used for noninvasive assessment of proliferation in pulmonary nodules, showing FLT uptake specificity for the differential diagnosis of pulmonary nodules [17]. More targeted approaches to studying small cell lung carcinomas are made by using 6-(18)F-fluoro-phenylalanine ([(18) F]FDOPA), as dihydroxyphenylalanine accumulates in tumors of neuroendocrine origin. Neuroendocrine immunohistochemical markers were used in evaluating [(18)F]FDOPA uptake in these patients, in comparison with standard FDG uptake, but no clear relation was proven between the radio-labeled marker and neuroendocrine IHC markers [18]. Another correlation was studied using the expression of P-glycoprotein (Pgp) as an immunomarker in low FDG PET uptake patients diagnosed with lung cancer, showing a correlation of Pgp and the degree of cell differentiation in adenocarcinomas, while FDG showed to be inversely correlated with cell differentiation [19]. More and more studies recently published, regarding nonsmall-cell lung cancer, show an interest in demonstrating correlations between FDG SUVmax values and immunohistochemical stains capable of offering insights into tumor proliferation and vascular status, such as Ki67 and CD34 [20]. Immunohistochemistry proved essential again in demonstrating a distinction between tuberculosis and non-small cell lung cancer nodules, in a recent study on 34 patients using (68)Ga-Alfatide II, which was shown to be a superior candidate to FDG in this matter [21].

However fruitful ongoing research in lung cancer seems, no less investigated was breast cancer. PET-CT imaging is a useful tool for the management of locally advanced breast cancer. Immunohistochemical staining for estrogen receptors, progesterone receptors, and HER2 status are routinely determined for their prognostic and therapeutic value in the management of patients. A clinical trial on 36 patients showed lower estrogen receptor expression in tumors with higher maximum SUV value [22]. Another clinical trial examined the relationship between FDG uptake and new vessel formation in twenty early breast carcinoma patients, using CD105 immunomarker [23]. 18F-fluoroestradiol PET and 18F-fluorodihydrotestosterone PET have been used, to such an extent, to prove the existence of androgen and estrogen receptors in breast cancer patients, the authors suggesting a very high cumulative sensitivity and sensibility for the examination, as to bypass immunohistochemical diagnosis altogether [24,25]. An interesting clinical trial, evaluating the relationships between FDG SUV values, tumor blood flow and glucose metabolism in breast cancer was conducted, showing an association of first-pass FDG uptake and angiogenesis, confirmed using CD34 immunomarkers, also noting the association between proliferation markers (using Ki-67) and tumor metabolism [26]. Human epidermal growth factor receptor status, alongside fluorescence in situ hybridization, in breast cancer patients, is currently the only one able to determine response predictions to trastuzumab emtansine therapy. This clinical trial argued that combining early metabolic assessment by FDG-PET/ CT uptake determinations may improve the understanding of tumor heterogeneity and selection of patients who will respond to the aforementioned therapy. Fifty-six patients were analyzed and immunohistochemical analysis was performed for HER2 status [27]. Finally, one trial performed a Ki-67 immuno-stain analysis, alongside SUV value determination, in breast cancer patients, showing different results in therapy responders versus non-responders who underwent preoperative systemic treatment [28].

\section{Discussion}

Pathology findings have been regarded for decades as the "golden standard" in the diagnosis and certification of various results, not only helping with clinical or surgical management but also being, at times, a determinant factor, with a considerable psychological impact, in the medicalpatient relationship. Of course, as in all fields of medicine, 
the science is not exact. Results vary dependent on the pathologist, quality of tissue samples and, not rarely, due to our limited knowledge of certain pathologies. It is understandable why people have critically questioned this elevated position in the past [29,30]. Efforts to outline and enhance these occasional diagnostic uncertainties have been made, over the years, through electron microscopy, immunohistochemistry, and molecular genetic tests, to name a few [31]. Multidisciplinary advances, attempting to integrate clinical and radiological information, only seem to underline the importance of histological data [32]. A more clinically feasible approach for disease identification and diagnostic judgment, besides pathology reports, may reside in enhancing the characteristics and limitations of PET-CT scans, as vectors for non-invasive diagnosis. As such, numerous endeavors are being made to establish correlations between PET-CT scans and histopathology, and more so with immunohistochemical ancillary tests. Nonetheless, until this hybrid imaging technique, of great value, will have proven itself in the future as a viable alternative in disease certification, it will still remain subject to its microscopic examination counterpart.

\section{Recent clinical trials}

Our efforts to make an accurate portrayal of current clinical trials that benefited from the practice of histopathologic and immunohistochemical certified PET-CT scans, revealed various results, ranging from trials which involved the study of vulnerable atheromas, neurodegenerative and inflammatory diseases to various types of cancer.

As shown, not only were there numerous IHC stains used, but also a number of various radio-labeled molecules, leading us away from the FDG standard and more towards specified and disease-oriented markers. One of the most used IHC antibodies was $\mathrm{Ki}-67$, which was shown to have a variable degree of correlation depending on the type of tumour assessed and its degree of differentiation. Other IHC markers, such as those used for vascular identification were also slightly more frequent. Recently, studies concerning new tumor vascular receptors have also been targeted as potential new markers for malignancy status [33]. The most studied types of neoplasms were those of breast and lung origin, which is of no surprise given the fact that these hold the first two places (with 2.09 million cases each) in the global cancer incidence of last year, lung cancer still being the first cause of cancer-related deaths worldwide [34].

In the course of 19 years, it may seem that 27 clinical trials may not be such a large number, taking into account the growing number of original articles published every year. One must consider, in this regard, the various aspects concerning the high costs of PET-CT examinations, as well as non-standard IHC stains used in research, in addition to running the clinical trials alone; more so even for prospective trials. Some trials used a number of other methods of confirming their results, showing the need for stronger multilateral evidence or perhaps a growing trend in histopathology alternatives. The strength of the findings in some of the trials is questionable, some of them being conducted with small study populations as low as 4 patients.

We must acknowledge the fact that this review suffered from several limitations. We recognize our narrow search of only MEDLINE/ PubMed and SCOPUS indexed articles. Searches in EMBASE, Cochrane Library, OVID, Web of Science and other sources could have produced considerable more results. Furthermore, the use of other combination of MeSH terms referring to the same subject may have yielded different or more results, nevertheless, perhaps not to the detriment of our statements. Finally, we concede the fact that having an anatomic pathologist as the main writer and investigator may be a concern for biased opinions.

\section{Tumor metabolism oriented research}

In the previous subtitle, we already touched upon different clinical trials attempting to establish a correlation between PET-CT SUV values and an IHC marker for cell proliferation, namely KI-67, in various types of cancers. Our further investigation of this research topic revealed numerous articles on the matter. Alongside articles concerning breast [23,35], thyroid [36], prostate [37] and head and neck neoplasms [38], results showed an interest for tumors of neuroendocrine origin as well $[39,40]$. Low study population groups were examined for cervical neoplasms [41], endometrial adenocarcinomas [42] and hepatocellular carcinomas [43]. Concerning PET-CT IHC validation, it seems that this correlation holds particular interest. Although this correlation shows a promising start for PET-CT validation as a stand-alone diagnostic tool, results vary considerably on the matter, some studies showing a positive correlation [12,44,45], while others showing different outcomes [42,46,47]. A recent metaanalysis on this topic concluded, with some degree of limitation, that FDG uptake presents a moderate positive correlation with tumor cell proliferation. Gastrointestinal stromal tumors and thymic epithelial tumors showed the maximum correlation coefficient $(0.81)$, a poor correlation being found in head and neck, gastric, thyroid and malignant melanoma, and moderate correlation in all the other neoplasms studied [39].

Emerging disease validation through microRNAs MicroRNAs represent non-coding forms of RNA molecules, usually 20 to 25 nucleotides in length, that control gene expressions, acting as intermediaries within the cell. Their importance becomes clear as it seems that they have an altered expression in different diseases including cancer, myocardial infarction, hepatitis $\mathrm{C}$ and metabolic diseases, to name only a few. This happens through overand underexpression, giving rise to specific micro ARN patterns. As such, not only do they represent a potential candidate for therapies, but also powerful biological markers for disease detection. Even with growing numbers 
of studies being conducted to enrich our knowledge of their true potential, we still do not have a complete grasp of their molecular mechanisms in gene regulation [48].

Our initial search for clinical trials involving these new molecular markers in correlation with PET-CT tracer uptake found no relevant results suggesting their novelty in this particular area of research.

Further investigation involving articles without the "clinical trial" restriction lead to noteworthy information. For example, as a counterpart for the aforementioned study concerning $[18 \mathrm{~F}]$ flutemetamol PET highlighted $\mathrm{A} \beta$ plaques, one study has found the value of microRNA detection in cerebrospinal fluid of Alzheimer's disease patients where amyloid PET imaging has limitations [49]. Another recent study investigated the predictive role of microRNA-21 and microRNA-148a in 36 patients with esophageal adenocarcinoma, correlating results with histopathology, survival and FDG-PET scans [50]. Concerning the ongoing struggle to better diagnose pancreatic cancer, articles propose miR-223, and other microRNAs alongside other biological markers, as a useful indicator with a potential prediction for aggressiveness [51,52]. Addition of miR205 and miR-210 to CA-19-9 increases specificity to $91 \%$ and sensitivity to $100 \%$ of pancreatic cancer detection while showing a correlation with decreased survival [53]. In one review, an expert's opinion was that a combination of such biomarkers and imaging technologies will develop into standard practice in the future [54]. Emerging publications show the role of microRNAs ranging from miR-206 as a potential target for diagnosis and therapy, in head and neck squamous cell carcinomas, to the use of microRNAs as potential markers for rejection in cardiac transplantation [55,56]. Another article addressed the diagnostic capabilities of microRNAs, alongside multiple other diagnostic tests including immunohistochemistry and FDG-PET, in thyroid cancers, particularly in those difficult to differentiate by histopathology alone. They argued that a multimodal approach is still the most likely to offer an accurate diagnosis in this case [57]. Thyroid neoplasms are often subjects of debate concerning not only diagnosis but, in some cases, also from a postsurgery management standpoint of view [58]. Some studies suggest that circulating tumor cells hold an answer to better prognosis and follow-up [59]. These cancers hold the potential for high interest in research, as they are found to have high regional and global incidence $[59,60]$. Finally, concerning early breast cancer diagnosis, one publication proposed microRNAs as new diagnostic and therapeutic methods alongside PET-CT and other imaging techniques [61].

Adding to their high specificity in diagnosing disease, especially when combined with clinical data and other proven types of biomarkers, microRNAs also require very small amounts of biological material and can be extracted from various sources including plasma, serum, fine-needle aspirates, pancreatic juice or saliva. They remain preserved in formalin-fixed or deep-frozen tissues as well [53].

This combination of properties, along with ongoing research beginning to emerge as proof of their feasibility in disease diagnosis, allow us to make the assumption that in the near future they may become a potential reliable candidate for PET-CT validation studies.

\section{Conclusions}

Our overview of the selected studies showed that histopathologic findings and IHC were invaluable for their completion and for a proper determination of the validity of their initial hypothesis.

Studies concerning Ki-67 correlation seem to be a worthwhile endeavor, considering its prognostic value, although for a limited number of neoplastic diseases.

The combination of microARN properties, alongside with ongoing research beginning to emerge as proof of their feasibility in disease diagnosis, allow us to make the assumption that in the near future they may become a potential reliable candidate for PET-CT validation studies.

\section{Acknowledgments}

This study received an internal grant awarded by the Iuliu Hatieganu University of Medicine and Pharmacy, Cluj-Napoca, Romania (contract no. 1680/25/19.01.2018), based on Ph.D. contract no. 4115/01.10.2017.

\section{References}

1. Piciu D. Nuclear Endocrinology. 2nd ed. Springer International Publishing; 2017: pp. 43-46.

2. Menezes LJ, Kotze CW, Agu O, Richards T, Brookes J, Goh VJ, et al. Investigating vulnerable atheroma using combined (18)F-FDG PET/CT angiography of carotid plaque with immunohistochemical validation. J Nucl Med. 2011;52:1698-1703.

3. Beach TG, Thal DR, Zanette M, Smith A, Buckley C. Detection of Striatal Amyloid Plaques with [18F] flutemetamol: Validation with Postmortem Histopathology. J Alzheimers Dis. 2016;52:863-873.

4. Chae SY, Choi CM, Shim TS, Park Y, Park CS, Lee HS, et al. Exploratory Clinical Investigation of (4S)-4-(3-18FFluoropropyl)-L-Glutamate PET of Inflammatory and Infectious Lesions. J Nucl Med. 2016;57:67-69.

5. Antwi K, Fani M, Nicolas G, Rottenburger C, Heye T, Reubi JC, et al. Localization of Hidden Insulinomas with ${ }^{68}$ Ga-DOTA-Exendin-4 PET/CT: A Pilot Study. J Nucl Med. 2015;56:1075-1078.

6. Lansoy-Kuhn C, Picquenot JM, Edet-Sanson A, Mechken F, Laberge-Le Couteulx S, Cornic M, et al. Relationship between the immunohistochemistry of the primary tumour and 18F-FDG-PET/CT at recurrence in patients with welldifferentiated thyroid carcinoma. Nucl Med Commun. 2013;34:340-346.

7. Mena E, Turkbey B, Mani H, Adler S, Valera VA, Bernardo $\mathrm{M}$, et al. 11C-Acetate PET/CT in localized prostate cancer: a 
study with MRI and histopathologic correlation. J Nucl Med. 2012;53:538-545.

8. de Oliveira Costa R, Hallack Neto A, Siqueira S, Lage LA de PC, de Paula HM, Coutinho AM, et al. Interim fluorine-18 fluorodeoxyglucose PET-computed tomography and cell of origin by immunohistochemistry predicts progression-free and overall survival in diffuse large B-cell lymphoma patients in the rituximab era. Nucl Med Commun. 2016;37:1095-1101.

9. Tian M, Zhang H, Nakasone Y, Mogi K, Endo K. Expression of Glut-1 and Glut-3 in untreated oral squamous cell carcinoma compared with FDG accumulation in a PET study. Eur J Nucl Med Mol Imaging. 2004;31:5-12.

10. Zhao Z, Yoshida Y, Kurokawa T, Kiyono Y, Mori T, Okazawa H. 18F-FES and 18F-FDG PET for differential diagnosis and quantitative evaluation of mesenchymal uterine tumors: correlation with immunohistochemical analysis. J Nucl Med. 2013;54:499-506.

11. Yamamoto $\mathrm{Y}$, Kameyama R, Izuishi K, Takebayashi R, Hagiike M, Asakura M, et al. Detection of colorectal cancer using ${ }^{18}$ F-FLT PET: comparison with ${ }^{18}$ F-FDG PET. Nucl Med Commun. 2009;30:841-845.

12. Riedl CC, Akhurst T, Larson S, Stanziale SF, Tuorto S, Bhargava A, et al. 18F-FDG PET scanning correlates with tissue markers of poor prognosis and predicts mortality for patients after liver resection for colorectal metastases. J Nucl Med. 2007;48:771-775.

13. Nakamura K, Kodama J, Okumura Y, Hongo A, Kanazawa S, Hiramatsu Y. The SUVmax of 18F-FDG PET correlates with histological grade in endometrial cancer. Int J Gynecol Cancer. 2010;20:110-115.

14. $\mathrm{Hu} \mathrm{M}$, Xing $\mathrm{L}$, Mu D, Yang W, Yang G, Kong L, et al. Hypoxia imaging with 18F-fluoroerythronitroimidazole integrated PET/CT and immunohistochemical studies in nonsmall cell lung cancer. Clin Nucl Med. 2013;38:591-596.

15. Baek S, Choi CM, Ahn SH, Lee JW, Gong G, Ryu JS, et al. Exploratory clinical trial of (4S)-4-(3-[18F]fluoropropyl)L-glutamate for imaging $\mathrm{xC}$ - transporter using positron emission tomography in patients with non-small cell lung or breast cancer. Clin Cancer Res. 2012;18:5427-5437.

16. Yang W, Zhang Y, Fu Z, Yu J, Sun X, Mu D, et al. Imaging of proliferation with 18F-FLT PET/CT versus 18F-FDG PET/CT in non-small-cell lung cancer. Eur J Nucl Med Mol Imaging. 2010;37:1291-1299.

17. BuckAK, Schirrmeister H, Hetzel M, Von Der Heide M, Halter G, Glatting G, et al. 3-deoxy-3-[(18)F]fluorothymidinepositron emission tomography for noninvasive assessment of proliferation in pulmonary nodules. Cancer Res. 2002;62:3331-3334.

18. Jacob T, Grahek D, Younsi N, Kerrou K, Aide N, Montravers F, et al. Positron emission tomography with [(18)F]FDOPA and [(18)F]FDG in the imaging of small cell lung carcinoma: preliminary results. Eur $\mathrm{J}$ Nucl Med Mol Imaging. 2003;30:1266-1269.

19. Higashi K, Ueda Y, Ikeda R, Kodama Y, Guo J, Matsunari $\mathrm{I}$, et al. P-glycoprotein expression is associated with FDG uptake and cell differentiation in patients with untreated lung cancer. Nucl Med Commun. 2004;25:19-27.
20. Han B, Lin S, Yu LJ, Wang RZ, Wang YY. Correlation of ${ }^{18}$ F-FDG PET activity with expressions of survivin, Ki67, and CD34 in non-small-cell lung cancer. Nucl Med Commun. 2009;30:831-837.

21. Kang F, Wang S, Tian F, Zhao M, Zhang M, Wang Z, et al. Comparing the Diagnostic Potential of 68Ga-Alfatide II and 18F-FDG in Differentiating Between Non-Small Cell Lung Cancer and Tuberculosis. J Nucl Med. 2016;57:672-677.

22. Osborne JR, Port E, Gonen M, Doane A, Yeung H, Gerald $\mathrm{W}$, et al. 18F-FDG PET of locally invasive breast cancer and association of estrogen receptor status with standardized uptake value: microarray and immunohistochemical analysis. J Nucl Med. 2010;51:543-550.

23. Groves AM, Shastry M, Rodriguez-Justo M, Malhotra A, Endozo R, Davidson T, et al. ${ }^{18} \mathrm{~F}-\mathrm{FDG}$ PET and biomarkers for tumour angiogenesis in early breast cancer. Eur $\mathrm{J}$ Nucl Med Mol Imaging. 2011;38:46-52.

24. Venema CM, Mammatas LH, Schröder CP, van Kruchten M, Apollonio G, Glaudemans AWJM, et al. Androgen and Estrogen Receptor Imaging in Metastatic Breast Cancer Patients as a Surrogate for Tissue Biopsies. J Nucl Med. 2017;58:1906-1912.

25. Gemignani ML, Patil S, Seshan VE, Sampson M, Humm JL, Lewis JS, et al. Feasibility and predictability of perioperative PET and estrogen receptor ligand in patients with invasive breast cancer. J Nucl Med. 2013;54:1697-1702.

26. Cochet A, Pigeonnat S, Khoury B, Vrigneaud JM, Touzery C, Berriolo-Riedinger A, et al. Evaluation of breast tumor blood flow with dynamic first-pass 18F-FDG PET/CT: comparison with angiogenesis markers and prognostic factors. J Nucl Med. 2012;53:512-520.

27. Gebhart G, Lamberts LE, Wimana Z, Garcia C, Emonts P, Ameye L, et al. Molecular imaging as a tool to investigate heterogeneity of advanced HER2-positive breast cancer and to predict patient outcome under trastuzumab emtansine (TDM1): the ZEPHIR trial. Ann Oncol. 2016;27:619-624.

28. Jacobs MA, Ouwerkerk R, Wolff AC, Gabrielson E, Warzecha $\mathrm{H}$, Jeter S, et al. Monitoring of neoadjuvant chemotherapy using multiparametric, ${ }^{23} \mathrm{Na}$ sodium MR, and multimodality (PET/CT/MRI) imaging in locally advanced breast cancer. Breast Cancer Res Treat. 2011;128:119-126.

29. Rorke LB. Pathologic diagnosis as the gold standard. Cancer. 1997;79:665-667.

30. Chari ST, Echelmeyer S. Can histopathology be the "Gold Standard" for diagnosing autoimmune pancreatitis? Gastroenterology. 2005;129:2118-2120.

31. Pfeifer JD, Hill DA, O'Sullivan MJ, Dehner LP. Diagnostic gold standard for soft tissue tumours: morphology or molecular genetics? Histopathology. 2000;37:485-500.

32. Wells AU. Histopathologic diagnosis in diffuse lung disease: an ailing gold standard. Am J Respir Crit Care Med. 2004;170:828-829.

33. Bonci EA, Irimie A, Buiga R, Cosmin Lisencu I, Iuliana Maja L, Piciu D. Follicle-stimulating hormone receptors: a new immunohistochemical marker in cancers? J BUON. 2017;22:1352-1359.

34. Bray F, Ferlay J, Soerjomataram I, Siegel RL, Torre LA, Jemal 
A. Global cancer statistics 2018: GLOBOCAN estimates of incidence and mortality worldwide for 36 cancers in 185 countries. CA Cancer J Clin. 2018;68:394-424.

35. Koo HR, Park JS, Kang KW, Han W, Park IA, Moon WK. Correlation between (18)F-FDG uptake on PET/CT and prognostic factors in triple-negative breast cancer. Eur Radiol. 2015;25:3314-3321.

36. Kaida H, Kawahara A, Hayakawa M, Hattori S, Kurata S, Fujimoto K, et al. The difference in relationship between 18F-FDG uptake and clinicopathological factors on thyroid, esophageal, and lung cancers. Nucl Med Commun. 2014;35:36-43.

37. Woythal N, Arsenic R, Kempkensteffen C, Miller K, Janssen JC, Huang $\mathrm{K}$, et al. Immunohistochemical Validation of PSMA Expression Measured by ${ }^{68} \mathrm{Ga}$-PSMA PET/CT in Primary Prostate Cancer. J Nucl Med. 2018;59:238-243.

38. Rasmussen GB, Vogelius IR, Rasmussen JH, Schumaker L, Ioffe $\mathrm{O}$, Cullen $\mathrm{K}$, et al. Immunohistochemical biomarkers and FDG uptake on PET/CT in head and neck squamous cell carcinoma. Acta Oncol. 2015;54:1408-1415.

39. Deng S, Zhang W, Zhang B, Chen YY, Li JH, Wu YW. Correlation between the Uptake of 18F-Fluorodeoxyglucose (18F-FDG) and the Expression of Proliferation-Associated Antigen Ki-67 in Cancer Patients: A Meta-Analysis. Tagliabue E, editor. PLoS One. 2015;10:e129028.

40. Johnbeck CB, Knigge U, Langer SW, Loft A, Berthelsen AK, Federspiel B, et al. Prognostic Value of 18F-FLT PET in Patients with Neuroendocrine Neoplasms: A Prospective Head-to-Head Comparison with 18F-FDG PET and Ki-67 in 100 Patients. J Nucl Med. 2016;57:1851-1857.

41. Surov A, Meyer HJ, Schob S, Höhn AK, Bremicker K, Exner $\mathrm{M}$, et al. Parameters of simultaneous ${ }^{18} \mathrm{~F}-\mathrm{FDG}-\mathrm{PET} / \mathrm{MRI}$ predict tumor stage and several histopathological features in uterine cervical cancer. Oncotarget. 2017;8:28285-28296.

42. Tsujikawa T, Yoshida Y, Kiyono Y, Kurokawa T, Kudo T, Fujibayashi $Y$, et al. Functional oestrogen receptor $\alpha$ imaging in endometrial carcinoma using $16 \alpha-\left[{ }^{18} \mathrm{~F}\right]$ fluoro- $17 \beta$-oestradiol PET. Eur J Nucl Med Mol Imaging. 2011;38:37-45.

43. Kitamura K, Hatano E, Higashi T, Narita M, Seo S, Nakamoto Y, et al. Proliferative activity in hepatocellular carcinoma is closely correlated with glucose metabolism but not angiogenesis. J Hepatol. 2011;55:846-857.

44. Cher LM, Murone C, Lawrentschuk N, Ramdave S, Papenfuss A, Hannah A, et al. Correlation of hypoxic cell fraction and angiogenesis with glucose metabolic rate in gliomas using 18F-fluoromisonidazole, 18F-FDG PET, and immunohistochemical studies. J Nucl Med. 2006;47:410-418.

45. Takenaka T, Yano T, Ito K, Morodomi Y, Miura N, Kawano $\mathrm{D}$, et al. Biological significance of the maximum standardized uptake values on positron emission tomography in non-small cell lung cancer. J Surg Oncol. 2009;100:688-692.

46. Buchmann I, Vogg AT, Glatting G, Schultheiss S, Möller $\mathrm{P}$, Leithäuser $\mathrm{F}$, et al. [18F]5-fluoro-2-deoxyuridine-PET for imaging of malignant tumors and for measuring tissue proliferation. Cancer Biother Radiopharm. 2003;18:327-337.

47. Westerterp M, Sloof GW, Hoekstra OS, Ten Kate FJ, Meijer GA, Reitsma JB, et al. 18FDG uptake in oesophageal adenocarcinoma: linking biology and outcome. J Cancer Res Clin Oncol. 2008;134:227-236.

48. Christopher AF, Kaur RP, Kaur G, Kaur A, Gupta V, Bansal P. MicroRNA therapeutics: Discovering novel targets and developing specific therapy. Perspect Clin Res. 2016;7:6874.

49. Lusardi TA, Phillips JI, Wiedrick JT, Harrington CA, Lind B, Lapidus JA, et al. MicroRNAs in Human Cerebrospinal Fluid as Biomarkers for Alzheimer's Disease. J Alzheimers Dis. 2017;55:1223-1233.

50. Ilhan-Mutlu A, Tezcan G, Schoppmann SF, Preusser M, Spyridoula K, Karanikas G, et al. microRNA-21 expression is elevated in esophageal adenocarcinoma after neoadjuvant chemotherapy. Cancer Invest. 2015;33:246-250.

51. Komatsu S, Ichikawa D, Miyamae M, Kawaguchi T, Morimura R, Hirajima S, et al. Malignant potential in pancreatic neoplasm; new insights provided by circulating miR-223 in plasma. Expert Opin Biol Ther. 2015;15:773785.

52. Laeseke PF, Chen R, Jeffrey RB, Brentnall TA, Willmann JK. Combining in Vitro Diagnostics with in Vivo Imaging for Earlier Detection of Pancreatic Ductal Adenocarcinoma: Challenges and Solutions. Radiology. 2015;277:644-661.

53. Frič P, Škrha J, Šedo A, Zima T, Bušek P, Kmochová $\mathrm{K}$, et al. Early detection of pancreatic cancer: impact of high-resolution imaging methods and biomarkers. Eur $\mathrm{J}$ Gastroenterol Hepatol. 2016;28:e33-e43.

54. Zhang Q, Chen S, Zeng L, Chen Y, Lian G, Qian C, et al. New developments in the early diagnosis of pancreatic cancer. Expert Rev Gastroenterol Hepatol. 2017;11:149-156.

55. Liu F, Zhao X, Qian Y, Zhang J, Zhang Y, Yin R. MiR206 inhibits Head and neck squamous cell carcinoma cell progression by targeting HDAC6 via PTEN/AKT/mTOR pathway. Biomed Pharmacother. 2017;96:229-237.

56. Crespo-Leiro MG, Barge-Caballero G, Couto-Mallon D. Noninvasive monitoring of acute and chronic rejection in heart transplantation. Curr Opin Cardiol. 2017 Mar 16. doi: 10.1097/HCO.0000000000000400. [Epub ahead of print]

57. de Koster EJ, de Geus-Oei LF, Dekkers OM, van Engenvan Grunsven I, Hamming J, Corssmit EPM, et al. Diagnostic Utility of Molecular and Imaging Biomarkers in Cytological Indeterminate Thyroid Nodules. Endocr Rev. 2018;39:154-191.

58. Muntean V, Domsa I, Zolog A, Piciu D, Fabian O, Bosu R, et al. Incidental papillary thyroid microcarcinoma: is completion surgery required? Chirurgia (Bucur). 2013;108:490-497.

59. Bădulescu IC, Bărbuş E, Piciu D. Circulating tumor cells in thyroid carcinoma - the prognostic role of this biomarker. Review of the literature. Clujul Med. 2017;90:256-261.

60. Piciu D, Irimie A, Piciu A. Investigation of thyroid carcinoma over 40 years, using the database of the Ion Chiricuta Institute of Oncology Cluj-Napoca. J BUON. 2014;19:524-529.

61. Jafari SH, Saadatpour Z, Salmaninejad A, Momeni F, Mokhtari M, Nahand JS, et al. Breast cancer diagnosis: Imaging techniques and biochemical markers. J Cell Physiol. 2018;233:5200-5213. 